\title{
Role of radiotherapy in combination with chemotherapy, targeted therapy, and immunotherapy in the management of pancreatic cancer
}

\author{
Andrew Sharabi • Joseph Herman • Vivian Weiss • \\ Daniel Laheru • Richard Tuli
}

Received: 8 August 2013 / Accepted: 4 September 2013 /Published online: 31 October 2013

(C) Springer-Verlag Berlin Heidelberg 2013

\begin{abstract}
Currently, pancreatic ductal adenocarcinoma (PDAC) remains a highly lethal disease despite advances in multimodality therapy. The majority of patients with PDAC present with locally advanced or metastatic disease, and additional therapies or treatment paradigms are needed to improve progression-free survival and overall survival. Alternate therapies, such as a nanoparticle albumin-bound form of paclitaxel, epidermal growth factor receptor (EGFR) inhibitors, vascular endothelial growth factor (VEGF) inhibitors, and poly(ADP-ribose) polymerase (PARP) $1 / 2$ inhibitors have been investigated for potential synergy with systemic agents in PDAC. Here, we review the current status of radiotherapy combined with systemic therapies for pancreatic cancer, with a focus on these targeted agents, novel immunotherapies, and discussion of the Abscopal effect.
\end{abstract}

Keywords Pancreatic cancer · PARP · Immunotherapy · Stereotactic $\cdot$ Radiation $\cdot$ Abscopal

\footnotetext{
A. Sharabi $(\bowtie) \cdot J$. Herman

Department of Radiation Oncology and Molecular Radiation Sciences, Sidney Kimmel Comprehensive Cancer Center, Johns Hopkins University School of Medicine, Baltimore, MD, USA e-mail: asharab2@jhmi.edu

V. Weiss

Department of Pathology, Microbiology, and Immunology, Vanderbilt University Medical Center, Nashville, TN, USA

D. Laheru

Department of Medical Oncology, Skip Viragh Center for Pancreatic Cancer Research and Patient Care, Sidney Kimmel Comprehensive Cancer Center at Johns Hopkins, Baltimore, MD, USA

R. Tuli

Department of Radiation Oncology, Samuel Oschin Comprehensive Cancer Institute, Cedars-Sinai Medical Center, Los Angeles, CA, USA
}

\section{Current systemic and targeted therapies with or without radiation therapy}

Chemotherapeutic agents

The majority of patients with pancreatic ductal adenocarcinoma (PDAC) present with either metastatic (40-50\%) or locally advanced (30-40\%) disease. Initial sites of metastases include the liver, peritoneum, and lung. Whereas important progress has been made in understanding the biology of PDAC, these findings have translated into only incremental gains in terms of clinical outcome. Treatment efficacy is primarily limited by drug resistance often in combination with significant treatment-related toxicity. Herein, we review current systemic and targeted agents for pancreatic cancer and then focus on novel agents and immunotherapies with specific discussion on interactions with radiation.

Historically, 5-flourouracil (5-FU)-based regimens represented the backbone treatment for PDAC with objective response rates and median overall survival rates of up to $9 \%$ and 6 months, respectively [1], until gemcitabine was validated in a landmark study by Burris et al. [2]. Treatment with gemcitabine led to a statistically significant improvement in clinical benefit response (CBR) and median overall survival (5.7 vs. 4.4 months, $P=0.0025$ ) when compared to bolus 5FU in patients with advanced PDAC. Although gemcitabine became the standard single-agent chemotherapeutic agent of choice for patients with advanced disease, the improvement has been modest. For example, the final results of the adjuvant trials, Radiation Therapy Oncology Group (RTOG) 9704 (5$\mathrm{FU}$, radiation with gemcitabine or 5-FU) and ESPAC-3 (5-FU or gemcitabine for 6 months), failed to show a significant difference between chemotherapeutic regimens $[3,4]$. In a phase III study, Cunningham et al. [5] compared gemcitabine 
with or without capecitabine in patients with advanced PDAC. The addition of capecitabine significantly improved objective response rate (19.1 vs. $12.4 \% ; P=0.034)$ and progressionfree survival [hazard ratio $(\mathrm{HR})=0.78 ; 95 \%$ confidence interval $(\mathrm{CI}), 0.66$ to $0.93 ; P=0.004]$. However, there was only a trend toward improved overall survival (OS) $(\mathrm{HR}=0.86 ; 95 \%$ CI, 0.72 to $1.02 ; P=0.08)$ compared with gemcitabine alone. These modest results have spurred the investigation of combination chemotherapies and novel therapeutic approaches.

One such approach is a nanoparticle albumin-bound form of paclitaxel (nab-paclitaxel; Abraxane). Abraxane was recently combined with gemcitabine in a phase I/II trial for patients with metastatic disease. Von Hoff et al. [6] reported excellent outcomes with a median OS of 12 months, objective response rate of $48 \%$, and PFS of 7.9 months. Results of the phase III MPACT trial comparing gemcitabine and Abraxane to gemcitabine alone were recently presented at GI ASCO 2013 and showed that the median overall survival was 8.5 months for the combination arm compared to 6.7 for gemcitabine alone (HR=0.72; $P=0.000015)$. Additionally, the time to treatment failure was prolonged significantly with this combination from 3.5 to 5.1 months $(\mathrm{HR}=0.70 ; P<0.0001)$. Positive results were also seen in the randomized phase III trial by Conroy et al. [7] who compared 5-fluorouracil, folic acid, irinotecan, and oxaliplatin (FOLFIRINOX) to single-agent gemcitabine in patients with metastatic PDAC. In addition to excluding patients with locally advanced, nonmetastatic disease, stringent inclusion criteria included no evidence of hyperbilirubinemia and good performance status [Eastern Cooperative Oncology Group (ECOG), PS $\leq 1]$. FOLFIRINOX led to an improvement in median OS (11.1 vs. 6.8 months; $\mathrm{HR}=0.57, P<0.001$ ), objective response rate ( 31.6 vs. $9.4 \%, P<0.001$ ), and progressionfree survival (6.4 vs. 3.3 months; $\mathrm{HR}=0.47, P<0.001$ ). However, toxicities associated with the FOLFIRINOX regimen were not insignificant. Rates of grade 3 and 4 neutropenia (45.7 vs. $21 \%, P<0.001)$ and febrile neutropenia (5.4 vs. $1.2 \%$, $P=0.03$ ) were more frequent in the combination arm and required the use of granulocyte colony-stimulating factor (GCSF $)$ in 42.5 versus $5.3 \%(P<0.001)$. Also, the incidence of grade 3 and 4 thrombocytopenia, diarrhea, and sensory neuropathy was significantly greater in the FOLFIRINOX group. The treatment-associated mortality was, however, low with only one toxic death reported in each arm. Despite these toxicities, patient reported that the quality of life was improved in the FOLFIRINOX arm when compared to gemcitabine alone. The trial included primarily body/tail lesions; thus, it is unclear if this regimen will be as efficacious in the head of pancreas tumors. Nevertheless, FOLFIRINOX is now considered the standard of care for metastatic pancreatic cancer, and it is being evaluated in the borderline (Alliance A021101), unresectable (RTOG 1102), and adjuvant settings. Extrapolation of these multiagent regimens to the nonmetastatic setting has prompted some to examine the potential role of concurrent and/or sequential radiotherapy with FOLFIRINOX or gemcitabine/Abraxane.

Targeting epidermal growth factor receptor

Alternate therapies, such as targeted agents, have also been investigated to assess potential synergy with systemic agents in PDAC. Epidermal growth factors and their corresponding receptors [epidermal growth factor receptor (EGFR)] have been identified as drivers of tumor proliferation and metastatic progression. Correspondingly, the majority of pancreatic tumors have been shown to overexpress EGFR [8]. Based on this rationale, inhibitors of EGFR have been investigated in PDAC. Erlotinib, a small molecule inhibitor of the EGFR, led to improved outcomes when combined with gemcitabine. In a randomized, phase III double-blind study, Moore et al. [9] reported that overall survival was significantly prolonged with the addition of erlotinib to gemcitabine with a HR of $0.82(95 \%$ CI, 0.69 to $0.99 ; P=0.038$, median 6.24 vs. 5.91 months). One-year survival was also greater with erlotinib plus gemcitabine (23 vs. $17 \% ; P=0.023$ ), as was progression-free survival (HR of $0.77 ; 95 \% \mathrm{CI}, 0.64$ to $0.92 ; P=0.004$ ) [9]. Although this benefit seems incremental, it is interesting to note that for the subset of patients who developed erlotinib-related skin toxicity grade of $\geq 2(36 \%)$, median survival was more than 5 months longer than patients without skin toxicity ( 10.5 vs. 5.3 months, $P<0.001)$. Despite these results, acceptance of erlotinib with gemcitabine as a standard of care option for advanced PDAC has been slow. On the other hand, a combination of erlotinib with chemoradiation may exploit possible complementary effects between these modalities. Preclinical data indicates that one mechanism by which tumor cells can become resistant to the apoptotic and antiproliferative effects of radiation therapy is through paracrine activation of EGFR by transforming growth factor alpha (TGF- $\alpha$ ), which is released after radiation exposure. EGFR inhibition has been demonstrated to block the anti-apoptotic effects of TGF- $\alpha$ shedding, thereby restoring the apoptotic response of tumor cells to radiation. These preclinical findings, along with the positive phase III trial of gemcitabine and erlotinib in the metastatic setting, provided a strong rationale to test erlotinib combined with chemoradiation. A phase II trial evaluating the safety and efficacy of concurrent capecitabine and radiation with erlotinib followed by gemcitabine and erlotinib maintenance therapy in patients with resected PDAC was recently reported [10]. A total of 48 patients with resected PDAC were treated with adjuvant erlotinib (100 mg daily) and capecitabine $\left(800 \mathrm{mg} / \mathrm{m}^{2}\right.$ twice daily Monday-Friday) concurrently with intensity-modulated radiotherapy (50.4 Gy in 28 fractions) followed by 4 cycles of gemcitabine $\left(1,000 \mathrm{mg} / \mathrm{m}^{2}\right.$ on days 1,8 , and 15 every 28 days $)$ and erlotinib (100 mg daily). Eighty-five percent had nodal involvement, and $17 \%$ had positive resection margins. Median relapse-free survival (RFS) was 15.6 months (95\% CI, 13.4-17.9), and median 
survival was 24.4 months (95\% CI, 18.9-29.7). Similar to the study by Moore et al. [9], the development of dermatitis conferred improved RFS (HR $=0.27 ; P=0.009$ ). During chemoradiation and maintenance chemotherapy, rates of grade $3 / 4$ toxicity were $31 / 2 \%$ and $35 / 8 \%$, respectively. Bao et al. [11] reported on a similar study where patients received gemcitabine and erlotinib for 6 months without radiation. The median overall survival was similar (approximately 24 months); however, the local recurrence rate was significantly higher without radiation, despite the fact that all patients had negative margins at the time of enrollment. The local control benefit with the addition of radiation and improved RFS in patients who develop dermatitis suggests that erlotinib may also benefit select patients in the adjuvant setting. The cooperative RTOG 0848 study will further evaluate whether the addition of erlotinib to gemcitabine confers a survival benefit in patients receiving chemotherapy alone or with adjuvant chemoradiation.

Based on similar rationale, Philip et al. [12] investigated the addition of cetuximab, the monoclonal antibody to EGFR, to gemcitabine versus gemcitabine alone in patients with advanced PDAC. No significant difference was seen between the two arms of the study with respect to the median survival time (6.3 months for the gemcitabine plus cetuximab arm vs. 5.9 months for the gemcitabine alone arm; hazard ratio $=1.06$; $95 \% \mathrm{CI}, 0.91$ to $1.23 ; P=0.23$, one sided). Objective responses and progression-free survival were also similar in both arms of the study. Although time to treatment failure was longer in patients on gemcitabine plus cetuximab $(P=0.006)$, the difference in length of treatment was only 2 weeks longer in the combination arm. Other than clinical toxicities such as skin rash, attempts to correlate the expression of molecular targets (e.g., EGFR) to clinical outcome in hopes of prospectively identifying the subset of patients who may respond have been unsuccessful [13]. For example, expression of EGFR did not correlate to outcome in either study investigating the use of EGFR-targeted inhibitors. In a multicenter phase II study by Crane et al. [14], advanced PDAC patients were treated locally with 2 months of induction cetuximab, gemcitabine, and oxaliplatin, followed by concurrent capecitabine, cetuximab, and 50.4 Gy in 28 fractions to the gross tumor volume plus margin (without elective nodal irradiation). This was followed by maintenance gemcitabine and cetuximab therapy [14]. Median overall survival was an impressive 19.2 months $(95 \% \mathrm{CI}$, 14.2 to 24.2 months), and 1-, 2-, and 4-year actuarial overall survival rates were 66,25 , and $11 \%$, respectively. Grade $2 / 3$ gastrointestinal toxicities were $32 / 10 \%$, respectively. In this study, Smad4/Dpc4 mutational analysis was performed on pretreatment fine-needle aspiration samples and found to be correlated with patterns of recurrence. Interestingly, Dpc4mutant samples were more likely to recur distantly, whereas Dpc4 wild-type patients tended to recur locally $(P=0.016)$. The role of Smad4/Dpc4 as a predictor of recurrence will be further explored in RTOG 1201, a trial evaluating dose-escalated radiation therapy in patients with locally advanced pancreatic cancer [15]. Although the use of cetuximab and erlotinib have had limited benefit in the metastatic setting, these results suggest that the use of EGFR inhibitors sequentially or in combination with chemoradiation has shown promising outcomes with acceptable toxicity, thereby warranting further evaluation.

Targeting vascular endothelial growth factor

Antiangiogenic targets have also theoretically held great promise in the treatment of PDAC. Unfortunately, clinical results to date have been quite disappointing. A single-arm phase II study by Kindler et al. [16] investigated the addition of the vascular endothelial growth factor (VEGF) monoclonal antibody, bevacizumab $(10 \mathrm{mg} / \mathrm{kg})$, to gemcitabine in patients with advanced pancreatic cancer. Of the 52 patients enrolled, $21 \%$ had a partial response, while $46 \%$ had stable disease. Median overall survival was 8.8 months. Whereas outcomes were somewhat improved relative to historic controls, a large proportion of enrolled patients had excellent performance status (ECOG 0 and 1), which potentially influenced clinical outcomes and perhaps is also not a representative of the general PDAC population. As a follow-up to the results of this somewhat promising study, the CALGB conducted a phase III placebo-controlled trial with patients $(n=535)$ randomized to receive gemcitabine with or without bevacizumab [17]. Unfortunately, results did not corroborate previous studies with median overall survival rates of 5.8 and 5.9 months for the experimental and control arms, respectively. Similar results were seen with the small molecule tyrosine kinase inhibitor of VEGF receptor (VEGFR)-1, VEGFR-2, and VEGFR-3, axitinib. Whereas results from a randomized phase II trial of gemcitabine with or without axitinib in advanced PDAC patients favored the combination arm (median OS of 6.9 vs. 5.6 months, respectively [18]), the subsequent phase III study was halted after interim analysis showed no benefit in survival.

\section{Novel agents, immunotherapy, and checkpoint blockade}

\section{Poly(ADP-ribose) polymerase-1/2 inhibition}

Targeted inhibition of DNA repair proteins is another mechanism to further exploit the synergy between chemotherapeutic agents, such as gemcitabine and 5-FU, with radiation in PDAC. Inhibitors of the poly(ADP-ribose) polymerase (PARP) $1 / 2$ have shown excellent antitumor activity when used with other cytotoxic therapies, and targeting tumor cells with preexisting defects in double-strand DNA repair (BRCA mutant) may allow preferential killing of such tumor cells. The potential to exploit both of these strategies exists in PDAC. To date, however, the majority of data investigating PARP inhibition is in vitro and preclinical with a number of 
early phase clinical studies currently underway. Rajan et al. [19] reported a phase I study of olaparib with cisplatin and gemcitabine in patients with solid tumors. One of three patients with PDAC in this cohort had a PR following treatment, and he also interestingly harbored a BRCA2 mutation [19]. A phase II multi-institutional study is currently investigating gemcitabine and cisplatin with or without veliparib in patients with PDAC and a known BRCA/PALB2 mutation (NCT01585805). Our in vitro investigations with veliparib have shown it to be a potent sensitizer of radiation and gemcitabine in the treatment of $\mathrm{MiaPaCa}-2$ pancreatic tumors with dose enhancement factors of up to 2.4 and minimal cytotoxicity when cells were treated with veliparib alone (Tuli et al., in preparation). In vivo, treatment with a single dose of veliparib, radiation, or veliparib and radiation led to a significant tumor growth inhibition of 8,30 , and 39 days, respectively; survival at 30 days for these groups was 65,70 , and $100 \%$, while at 60 days, it was 0,0 , and $40 \%$, respectively. Furthermore, radiation-induced apoptosis was significantly enhanced by veliparib, suggesting a synergistic mechanism of interference with DNA repair. In vitro PAR levels correlated with levels of tumor apoptosis, suggesting its potential as a predictive clinical biomarker [20]. We have translated this promising data into a phase I study for patients with locally advanced PDAC investigating the maximum tolerated dose, safety, and toxicity of veliparib with full-dose gemcitabine and intensitymodulated radiotherapy. As evidenced by many of the negative randomized trials already discussed, it is critical that the preclinical evaluation of a new therapeutic strategy utilizes models that closely resemble contemporary clinical scenarios, so that highyield early phase clinical trials can be designed. This is particularly important for radiotherapy studies in PDAC where intensification of local therapy has the potential to improve not only local control but also survival. Unfortunately, radiation techniques applied to small animals are archaic, often single beam, and nonuniform, bearing little resemblance to the way patients are treated. In order to bridge this translational gap, our group has developed a novel preclinical pancreatic cancer research model using bioluminescence image-guided conformal irradiation of orthotopic xenograft tumors using the small animal radiation research platform (SARRP) [21]. This allows accurate three-dimensional targeting of tumors and sparing of surrounding normal tissues, thereby facilitating high-throughput investigation of novel molecular targeting agents with radiosensitizing potential $[22,23]$. As new targeted therapies are developed, it is imperative that preclinical platforms determine the optimal regimen prior to clinical implementation.

\section{Immunotherapeutic strategies}

Immunotherapy aims to provide the ideal cancer therapy through systemic, highly specific, and nontoxic antitumor activity. However, until very recently, most immunotherapy approaches have not proven to be very potent. Methods which enhance the induction and potency of the immune response are needed in order to continue the progress of immunotherapy in the clinic.

Development of an immune response is a complex and highly regulated process which involves interaction of multiple different cell types (Fig. 1). In a simplified example, an antigen-presenting cell (APC) such as a macrophage or dendritic cell (DC) captures an antigen which it then presents via MHC on the cell surface [24]. The APC then migrates back to a lymph node where it interacts with cluster of differentiation (CD) $4 \mathrm{~T}$ helper cells. The $\mathrm{T}$ cell receptor binds to the antigen presented on $\mathrm{MHC}$, and in the presence of proper co-stimulation, a $\mathrm{T}$ cell or $\mathrm{B}$ cell is then signaled to propagate an immune response resulting in the development of cytotoxic effector $\mathrm{T}$ cells or specific antibodies, respectively. There are classically two signals which are needed in order to induce a specific immune response: signal 1 is the antigen, and signal 2 is the costimulatory surface molecule. Professional APCs including DCs are the most potent antigen-presenting cells capable of inducing immune responses due to their high-antigen presentation capacity and high expression of co-stimulatory molecules and ability to secrete significant levels of co-stimulatory cytokines. The presence of these co-stimulatory molecules cannot be understated, as DCs, which do not express co-stimulatory molecules, can cause $\mathrm{T}$ cell anergy and can actively suppress the development of a specific immune response.

Fortunately for the host, one of the defining features of the immune system is central and peripheral self-tolerance. Central self-tolerance is initiated during development in part by selecting out $\mathrm{T}$ cells in the thymus which bind strongly to selfantigens. If these self-reactive $\mathrm{T}$ cells were released into the periphery, they would cause severe autoimmune disease which could be life threatening to the host. Unfortunately, cancer is a disease of autologous cells, and while exogenous factors such as carcinogens, mutagens, and viruses can cause cancer cells to express novel or foreign antigens, the host has already set up a significant self-tolerance to cancer cells, which is difficult to overcome, and could limit the potency of any immune response that is developed. Multiple tumor antigens have been identified in pancreatic cancer, including mucin MUC1, GA733 glycoproteins, ras peptide, EGFR, carcinoembryonic antigen (CEA), and mesothelin $[25,26]$. Methods to break tolerance to these tumor antigens will be critical to unlocking the potential of immunotherapy for pancreatic cancer.

Peripheral tolerance must also be overcome in order to generate a robust immune response to tumor antigens. Multiple mechanisms serve to maintain peripheral tolerance, including inhibitory cytokines, inhibitory cell surface molecules, and regulatory cell populations which function to dampen or block immune responses. T regulatory cells (Tregs) are one of the most powerful regulatory cell populations which 


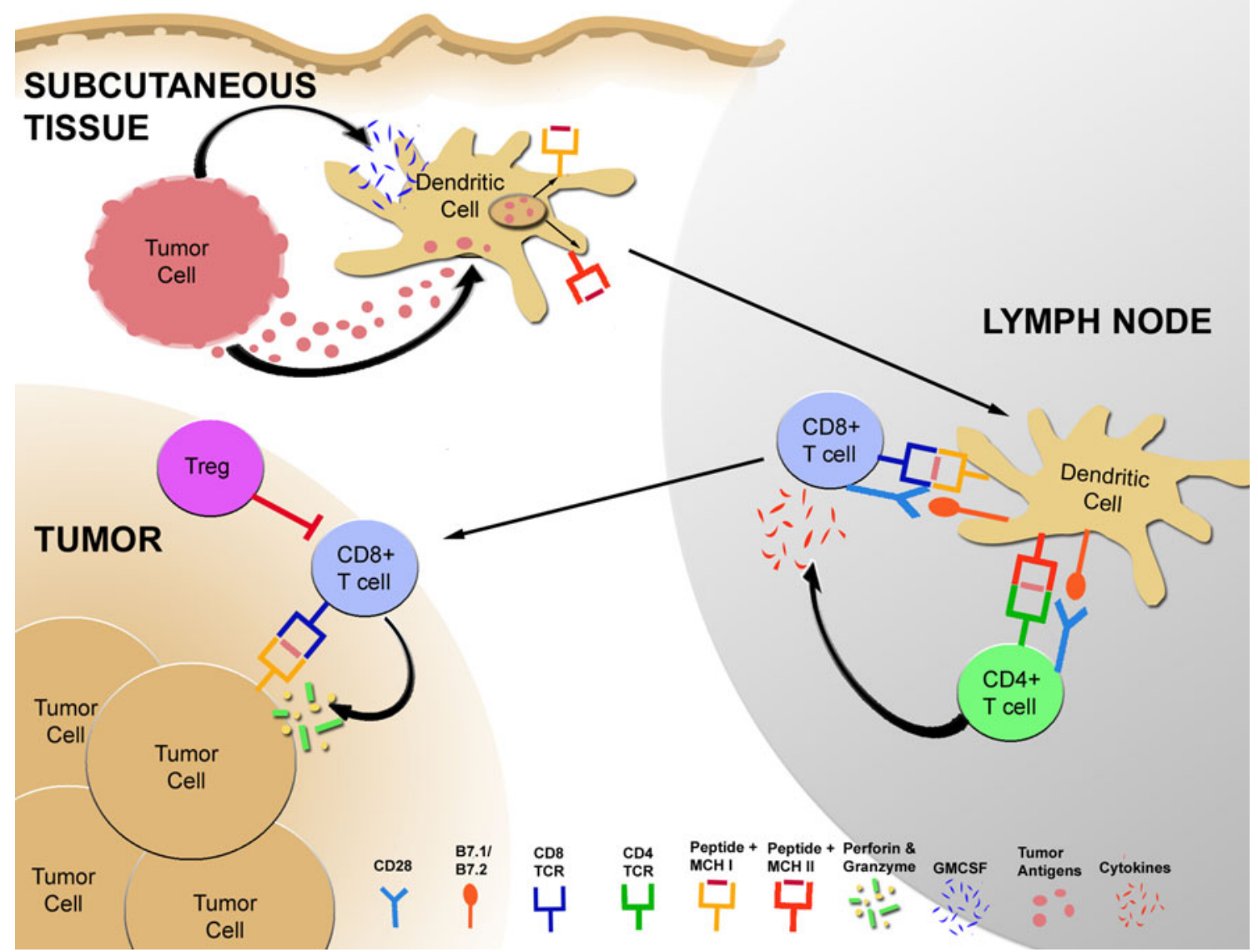

Fig. 1 Model of whole-cell vaccination. After injection of the tumor vaccine, the irradiated tumor cells express tumor antigens and GMCSF. The dendritic cells take up the antigen and migrate to the draining lymph node where they activate antigen-specific $\mathrm{CD} 4^{+}$and $\mathrm{CD} 8^{+} \mathrm{T}$ cells. The

can proliferate and traffic to peripheral sites to inhibit immune responses (Fig. 2). Strategies to inhibit or deplete Tregs are activated $\mathrm{CD}^{+} \mathrm{T}$ cells are then able to migrate to the tumor microenvironment and mediate tumor lysis. T regulatory cells in the tumor microenvironment lead to $\mathrm{CD} 8^{+} \mathrm{T}$ cell suppression

critical to the success of antitumor immunotherapy in the clinic $[27,28]$. One interesting clinically feasible strategy uses low-

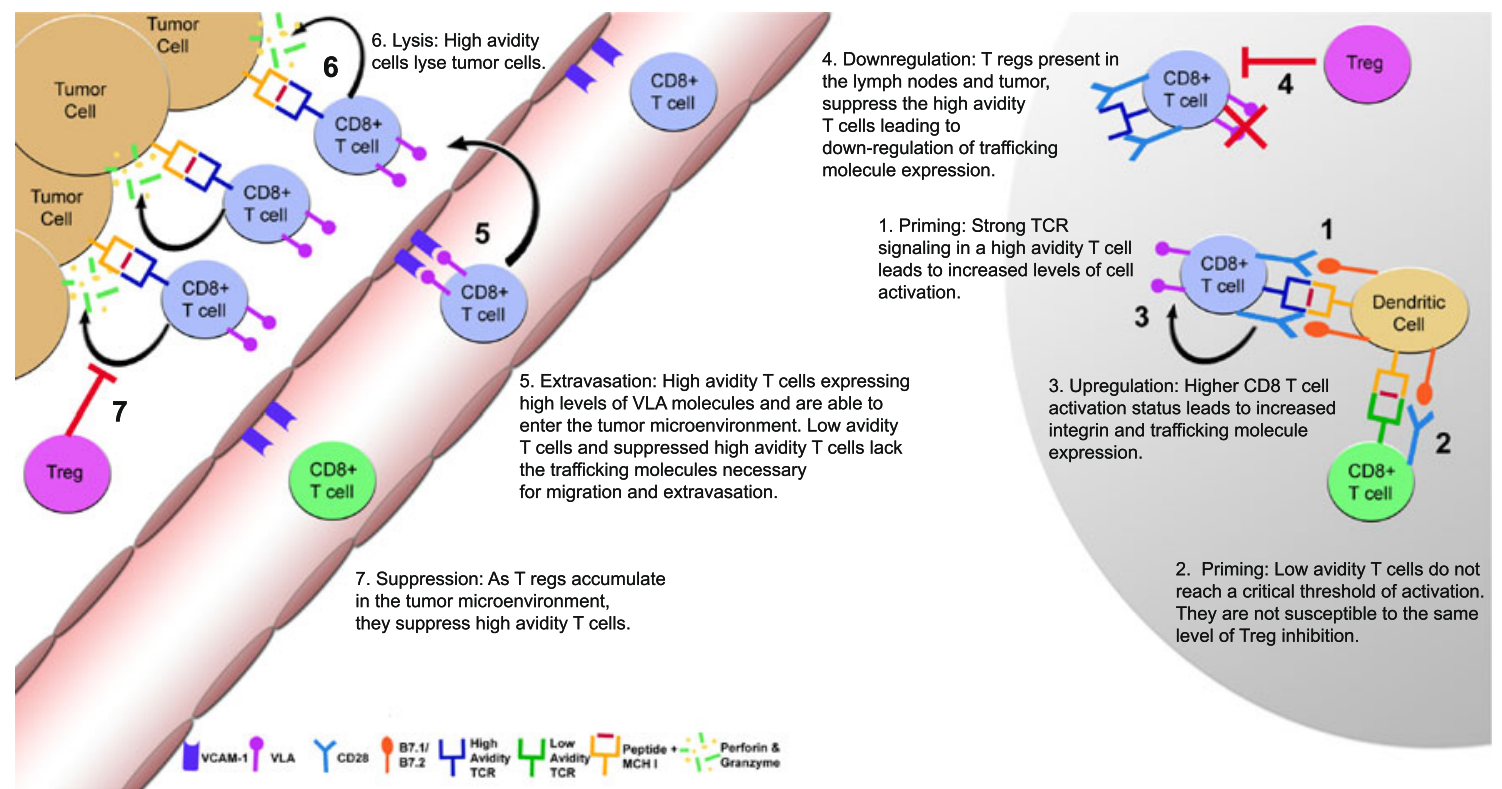

Fig. 2 High and low avidity tumor-specific T cell activation, trafficking, and suppression 
dose cyclophosphamide which has been shown to suppress Tregs and allow more effective induction of antitumor immune responses [29]. The presence of these powerful suppressor populations highlights the need for combinatorial immunotherapy strategies which stimulate an immune response while simultaneously blocking Tregs or immune checkpoints.

\section{Interferon alpha}

Multiple agents and techniques have been used clinically to attempt to promote anticancer immune responses against PDAC. One of the best studied agents is interferon alpha (IFN- $\alpha$ ), which is a pleiotropic cytokine with multiple functions including enhancing antiviral and antitumoral responses. IFN- $\alpha$ has been shown to enhance the differentiation and function of DCs and macrophages, promotes $\mathrm{CD} 8^{+}$memory responses, and also enhances the activity of natural killer (NK) cells [30-33]. Additionally, IFN- $\alpha$ modulates the activity of the proteosome to produce more immunogenic antigens and simultaneously upregulates MHC class I expression, resulting in an increased immunogenic antigen expression on the cell surface [34]. In the proper context, IFN- $\alpha$ can also be pro-apoptotic, resulting in radiosensitization as well as chemosensitization [35-37].

Given these potentially beneficial properties, IFN- $\alpha$ was studied in combination with chemoradiation for PDAC. A phase III randomized controlled trial (CapRI) compared 5FU/folic acid (FA) to 5-FU, cisplatin, IFN- $\alpha$ (3 million units three times a week), and radiation therapy (50.4 Gy in 28 fractions) in patients with resected PDAC [38]. In 2010, results from 110 patients with a minimum of 2 years of follow-up were presented [39]. Median survival of patients treated with 5-FU/FA was 28.5 months (95\% CI, 19.5, 38.6) versus 32.1 months (95\% CI, 22.8, 42.2) for patients treated with chemoradioimmunotherapy (CRI). The survival curves clearly separated, although did not reach a statistical significance. Interestingly, analysis of patients who responded to IFN- $\alpha$ with decreased T regulatory cells and increased NK cell-mediated cytotoxicity had significantly longer survival. Grade 3 or 4 toxicity was increased in the CRI group as compared to 5-FU/FA, although there was no reported difference in quality of life. Thus, this trial demonstrated a higher median overall survival with CRI, which was especially pronounced in the subset of patients who appeared to respond to IFN- $\alpha$. Of note, dose reduction was required in these patients, and prior studies from different groups reported significant gastrointestinal toxicities [39]. Due to these side effects, a phase II de-escalation trial (CapRI-2) was opened in 2009 [40] with the goal of maintaining clinical efficacy through the immunostimulatory properties of IFN- $\alpha$, while limiting toxicity by omitting cisplatin or radiotherapy in separate deescalation arms. The results of this study should provide an interesting insight into the effects of IFN- $\alpha$ immunotherapy with or without cisplatin chemotherapy or radiotherapy for resected PDAC.

\section{Whole cell and dendritic cell vaccines}

One of the main goals of cell based immunotherapy is to induce an effective antitumor response using the vaccinated cells as an antigenic and immunostimulatory source. One of the theoretical advantages to cell-based vaccines is the halflife of the cells and their ability to induce a multifaceted and robust immune response via cell-cell interactions with $\mathrm{T}$ cell and $\mathrm{B}$ cells. However, the widespread implementation of this approach has been hampered by logistical challenges, cost, and limited clinical efficacy. Recent efforts have focused on enhancing the potency of whole-cell and DC vaccines with the goal of increasing the magnitude and duration of the induced antitumor $\mathrm{T}$ cell responses. Interestingly, granulocyte-macrophage (GM) CSF is commonly used in conjunction with cell vaccines in attempt to boost the response using cytokine costimulation.

Results from a large single-institution phase II trial of a GM-CSF transfected allogeneic cell-based tumor vaccine for PDAC in combination with chemoradiation were recently reported by Lutz et al. [41]. Sixty patients with resected PDAC received immunotherapy consisting of $5 \times 10^{8}$ lethally irradiated GM-CSF-secreting allogeneic whole-cell tumor cell lines distributed among three lymph node regions. The first immunotherapy treatment was administered 8 to 10 weeks after resection, and patients then went on to receive adjuvant chemoradiation with 5-FU. Patients subsequently received up to a total of five whole-cell immunotherapy treatments after completion of chemoradiation. Median disease-free survival was 17.3 months (95\% CI, 14.6-22.8) with median survival of 24.8 months (95\% CI, 21.2-31.6), which correlates well when compared with historical outcomes for resected pancreatic cancer. This whole-cell vaccine was safe and well tolerated with no observed local or systemic dose-limiting toxicities. Interestingly, induction of mesothelin-specific $\mathrm{CD} 8^{+} \mathrm{T}$ cells in $\mathrm{HLA}-\mathrm{A} 1^{+}$and HLA-A2 ${ }^{+}$patients correlated with disease-free survival. This finding suggests that patients who responded to the immunotherapy with specific immune responses had improved outcomes. Further techniques to improve the potency and response rate of whole-cell immunotherapy are needed to improve the clinical efficacy of this new modality for treating PDAC. Interestingly, it was found that following standard chemoradiation (tumor bed and regional lymph nodes), it took several months to years for certain cell counts to reach pre-treatment levels. Another study showed that adjuvant CRT can cause lymphopenia which can have a negative effect on survival [42]. Based on this rationale, integration of focused stereotactic body radiation therapy (SBRT) to the tumor bed and vascular margin alone may result in less immunosuppression and potentially better outcomes 
$[43,44]$. Furthermore, since SBRT can be ablative, it can potentially enhance immune responses and release additional tumor-specific antigens by ablating any residual microscopic disease. This approach is currently being evaluated in conjunction with FOLFIRINOX in an adjuvant pancreatic cancer phase I study at Johns Hopkins (NCT01595321).

Retrospective data from Japan in a series of 16 patients treated with DCs and lymphokine-activated killer lymphocytes (LAKs) as a second line therapy has been reported [45]. In this review of 16 patients with refractory pancreatic cancer, 11 patients received immunotherapy combined with chemotherapy, and 6 patients received immunotherapy alone. Patients received either combined DC vaccination intravenously or intraperitoneally or injection of LAK. The DCs were primed with antigenic lysates from necrotic tumor cells or with peptides from CEA or MUC1. Patients received two $30 \times 10^{6}$ mature DCs loaded with necrotic tumor cells or peptides every 2 or 3 weeks. They reported a mean survival of 9.7 months and median survival of 9 months, which appeared to favor those patients who received DC vaccination over LAK vaccination. Wholecell and DC immunotherapy in combination with chemotherapy and/or radiation deserves further investigation in patients with locally advanced PDAC, as it is well tolerated and likely provides a benefit for the subset of patients with an induced immune response.

\section{Cytotoxic T lymphocyte antigen 4 and PD1 antibodies}

Immunostimulatory antibodies, such as those against cytotoxic T lymphocyte antigen 4 (CTLA-4) (ipilimumab), hold great promise in a wide variety of tumor types (Fig. 3). Given the potentially lethal effects of autoimmune cytotoxic $\mathrm{T}$ lymphocytes, there are powerful negative regulatory mechanisms which serve to dampen or shut down $\mathrm{T}$ cell responses. Both CTLA-4 and CD28 are present on the T cell surface, and both can bind co-stimulatory molecules B7-1 and B7-2 on APCs. However, while $\mathrm{CD} 28$ transmits a positive activating signal to T cells, binding of CTLA-4 transmits a powerful inhibitory signal to T cells which can block $\mathrm{T}$ cell activation. Indeed, one of the most powerful negative regulatory molecules on the cell surface of T cells is CTLA-4 [46]. Similarly, the PD1 receptor is an additional negative regulator present on the T cell surface [47]. Antibodies which function to block binding and subsequent downstream signaling of these negative regulators can result in unbridled $\mathrm{T}$ cell activation (inhibiting an inhibitor resulting in activation) and can even break self-tolerance, resulting in autoimmune reactions.

Results from a phase II trial of single-agent ipilimumab (anti-CTLA-4) for locally advanced or metastatic PDAC were reported in 2010 [48]. In this single-arm single-institution phase 2 trial, 27 patients with advanced pancreatic cancer received ipilimumab intravenously at $3.0 \mathrm{mg} / \mathrm{kg}$ every 3 weeks for a total of eight doses. There were no responders by their criterion, but there was one patient with a late delayed response with significant regression of primary disease and metastatic burden and improvement in performance status. Whereas these results show that ipilimumab at $3.0 \mathrm{mg} / \mathrm{kg} / \mathrm{dose}$ is ineffective for advanced PDAC, further investigation into combinatorial therapies, which provide appropriate tumor antigen with ipilimumab, is deserved, as are efforts to identify potential responders to such therapies.

\section{The abscopal effect}

Cytotoxic therapies, such as radiation, are known to inhibit the immune system. However, recent provocative evidence suggests that focused radiation can, in fact, stimulate an antitumor immune response. An abscopal effect is such a response outside the field of radiation treatment. This phenomenon was originally coined by Dr. R.H. Mole in 1953 as an effect of radiation away from the primary target or, in other words, an action at a distance [49]. Work by Silvia Formenti and colleagues from New York University has further characterized the abscopal effect [50, 51]. Demaria et al. found that the growth of a nonirradiated mammary carcinoma was impaired with the use of Flt3 ligand and radiation to a contralateral flank tumor [50]. The authors also provided evidence that this effect was T cell dependent, as it was not observed in T cell-deficient mice. However, other groups have implicated alternate mechanisms for the abscopal effect, including the p53 pathway [52]. Given the nonintuitive nature of this phenomenon, it is certainly possible that multiple complex mechanisms may be at play, including those mediated by any combination of cellular, cytokine, and transcription factors, which themselves may be dependent on radiation dose and fractionation schemas.

Nevertheless, radiation is known to induce inflammation, apoptosis, cell death, and release of tumor antigens [53], which would create an ideal local environment for induction of a potent immune response [50, 54-56]. An additional and possibly more subtle effect of radiation is damage to the endothelium, increasing permeability, and thus, access for cells of the immune system into the tumor microenvironment. Given these local effects, the mechanisms by which focused radiation can help to stimulate an immune response appear to be quite clear. Unfortunately, aggressive tumor cells have powerful mechanisms of suppressing the immune system including limiting expression of antigens, expression of inhibitory cell surface molecules, and secretion of inhibitory cytokines. Furthermore, due to the inherent self-tolerance of the immune system toward autologous cancer cells, the repertoire of circulating $\mathrm{T}$ cells which can bind avidly to self-tumor antigens may be limited. Thus, multiple synergistic techniques or combinatorial therapies would theoretically have a better 
Fig. 3 a APC-T cell interaction with CD28 and CTLA-4 on the T cell surface. B7 binds both CD28 which promotes $\mathrm{T}$ cell activation via immunoreceptor tyrosinebased activation motif (ITAM) and CTLA-4 which blocks T cell activation via immunoreceptor tyrosine-based inhibitory motif (ITIM). b In the presence of antiCTLA-4 antibody (ipilimumab), inhibitory signaling by CTLA- 4 is abrogated leading to unbridled $\mathrm{T}$ cell activation. Additional possible mechanism includes depletion of Tregs by anti-CTLA4. Further investigation is needed to elucidate the mechanisms by which anti-CTLA-4 functions to stimulate immune responses
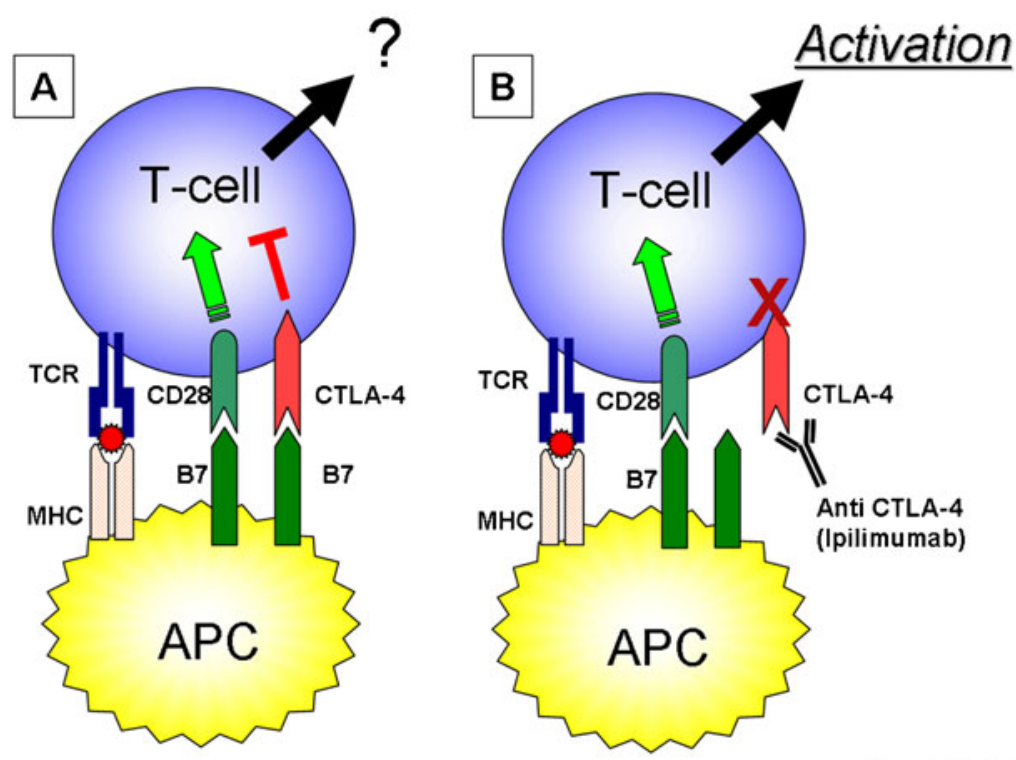

Sharabi et al. chance of activating the immune system and breaking selftolerance to PDAC cells.

An intriguing paper by Cao et al. [57] studied the effect of a sublethal dose of radiation on the efficacy of antitumor adoptive $\mathrm{T}$ cell transfer in a transgenic model of PDAC. In this study, the authors used Rip1-Tag2 mice which rapidly develop lethal pancreatic tumors due to the expression of the SV40 large $T$ antigen (Tag) oncogene under the control of the Rat insulin promoter (Rip1). The mice then received an adoptive transfer of Tag2 antigen-specific T cells with or without sublethal radiation with $6 \mathrm{~Gy}$. The authors reported that transfer of Tag-specific $\mathrm{T}$ cells alone did not result in a significant $\mathrm{T}$ cell infiltration into solid tumors and did not affect tumor growth or survival. However, in combination with 6 Gy of radiation, there was a significant lymphocyte infiltration into solid tumors which resulted in decreased tumor burden and improved survival. These important findings highlight the utility of radiation in generating an inflammatory and immunostimulatory tumor microenvironment, which synergizes with novel immunotherapies. Meng et al. [58] found a more robust immunogenic cytokine and chemokine profile, including upregulation of interferon beta, CXCL9, and CXCL-11, with radiation and veliparib as compared to radiation alone in mouse models of Her2 overexpressing P1048 pancreatic cancer cells. Moreover, when these pretreated pancreatic cancer cells were injected into mice, they induced HER-2-specific interferon gammaproducing T cells.

Blanquicett et al. [59] analyzed the effects of radiation with or without capecitabine and celecoxib in a xenograft model of irradiated and contralaterally shielded pancreatic tumors. Mice were injected bilaterally with BxPC-3 pancreatic cancer xenografts, and one flank received $10 \mathrm{~Gy}$ of radiation (2 Gy $\times$ five fractions), while the contralateral body and flank were shielded with lead. Capecitabine and/or celecoxib was administered and compared to mice treated with radiation alone. As expected, radiation alone reduced tumor size only in the irradiated xenografts, while capecitabine alone reduced tumor size in both the irradiated and nonirradiated tumors. However, when radiation was combined with capecitabine, there was a significant additional reduction in tumor size of the contralaterally shielded nonirradiated tumor when compared to capecitabine alone. This abscopal effect of radiation on the contralaterally shielded tumor was increased further when capecitabine and celecoxib were used in combination. Thus, these studies demonstrate a clear antitumor effect of radiation outside of the treated field when combined with chemotherapy and targeted therapies in preclinical models of PDAC. Given the high percentage of pancreatic cancer patients with metastatic disease at the time of diagnosis, harnessing and promoting the abscopal effects of radiation is an exciting new area which could potentially improve clinical outcomes.

\section{Conclusion and future directions}

PDAC remains a highly lethal disease despite advances in multimodality therapy, including surgery and radiation concurrent with aggressive systemic therapy regimens. The development of multiagent chemotherapy regimens, such as FOLFIRINOX, has improved outcomes and helps to control the rapid progression of this disease. However, additional strategies are needed to improve survival and mitigate the dose-limiting toxicities of current treatments. The use of novel small molecule-targeted agents, such as tyrosine kinase 
inhibitors or PARP inhibitors, in combination with chemotherapy and radiation is very promising and is a focus of active investigation.

Cytotoxic therapies such as chemotherapy and radiation are thought to suppress the immune system. However, emerging data now shows that cell death caused by cytotoxic therapies actually helps promote the development of a specific immune response. With focused radiotherapy, such as SBRT, the resulting cell death and inflammation could frame the ideal environment for antigen presentation and induction of an enhanced cytotoxic immune response. Furthermore, immunotherapy with monoclonal antibodies is gaining recognition as a useful modality with proven clinical efficacy in multiple different cancer subtypes. Due to the cytotoxic, inflammatory, and possible abscopal effects of radiation, it is our belief that combining these novel agents with strategic use of focused radiation (tumor plus a small margin) could lead to synergistic effects and improved clinical outcomes. Identification of the optimal radiation dose and fractionation schemes to maximize these effects should also be topics of further investigation.

Conflict of interest Andrew Sharabi, Joseph Herman, Vivian Weiss, Daniel Laheru, and Richard Tuli declare that they have no conflict of interest.

\section{References}

1. Van Rijswijk RE, Jeziorski K, Wagener DJ, Van Laethem JL, Reuse S, Baron B, Wils J, EORTC GastroIntestinal Tract Cancer Cooperative Group (2004) Weekly high-dose 5-fluorouracil and folinic acid in metastatic pancreatic carcinoma: a phase II study of the EORTC GastroIntestinal Tract Cancer Cooperative Group. Eur J Cancer 40(14):2077-2081

2. Burris HA 3rd et al (1997) Improvements in survival and clinical benefit with gemcitabine as first-line therapy for patients with advanced pancreas cancer: a randomized trial. J Clin Oncol 15(6): 2403-2413

3. Regine WF, Winter KA, Abrams RA, Safran H, Hoffman JP, Konski A, Benson AB, Macdonald JS, Kudrimoti MR, Fromm ML, Haddock MG, Schaefer P, Willett CG, Rich TA (2008) Fluorouracil vs gemcitabine chemotherapy before and after fluorouracil-based chemoradiation following resection of pancreatic adenocarcinoma: a randomized controlled trial. JAMA 299(9):1019-1026

4. Neoptolemos JP et al (2010) Adjuvant chemotherapy with fluorouracil plus folinic acid vs gemcitabine following pancreatic cancer resection: a randomized controlled trial. JAMA 304(10):1073-1081

5. Cunningham D, Chau I, Stocken DD, Valle JW, Smith D, Steward W, Harper PG, Dunn J, Tudur-Smith C, West J, Falk S, Crellin A, Adab F, Thompson J, Leonard P, Ostrowski J, Eatock M, Scheithauer W, Herrmann R, Neoptolemos JP (2009) Phase III randomized comparison of gemcitabine versus gemcitabine plus capecitabine in patients with advanced pancreatic cancer. J Clin Oncol 27(33):5513-5518, PMID: 19858379

6. Von Hoff DD et al (2011) Gemcitabine plus nab-paclitaxel is an active regimen in patients with advanced pancreatic cancer: a phase I/II trial. J Clin Oncol 29(34):4548-4554
7. Conroy T, Desseigne F, Ychou M et al (2011) FOLFIRINOX versus gemcitabine for metastatic pancreatic cancer. N Engl J Med 364(19): $1817-1825$

8. Tobita K, Kijima H, Dowaki S et al (2003) Epidermal growth factor receptor expression in human pancreatic cancer: Significance for liver metastasis. Int J Mol Med 11(3):305-309

9. Moore MJ, Goldstein D, Hamm J et al (2007) Erlotinib plus gemcitabine compared with gemcitabine alone in patients with advanced pancreatic cancer: a phase III trial of the National Cancer Institute of Canada Clinical Trials Group. J Clin Oncol 25(15):19601966

10. Herman JM, Fan KY, Wild AT et al (2013) Phase 2 study of erlotinib combined with adjuvant chemoradiation and chemotherapy in patients with resectable pancreatic cancer. Int J Radiat Oncol Biol Phys 86(4):678-685, PMID: 23773391

11. Bao PQ, Ramanathan RK, Krasinkas A et al (2011) Phase II study of gemcitabine and erlotinib as adjuvant therapy for patients with resected pancreatic cancer. Ann Surg Oncol 18(4):1122-1129

12. Philip PA, Benedetti J, Corless CL et al (2010) Phase III study comparing gemcitabine plus cetuximab versus gemcitabine in patients with advanced pancreatic adenocarcinoma: Southwest Oncology Group-directed intergroup trial S0205. J Clin Oncol 28(22):3605-3610

13. da Cunha SG, Dhani N, Tu D, Chin K, Ludkovski O, Kamel-Reid S, Squire J, Parulekar W, Moore MJ, Tsao MS (2010) Molecular predictors of outcome in a phase 3 study of gemcitabine and erlotinib therapy in patients with advanced pancreatic cancer: National Cancer Institute of Canada Clinical Trials Group Study PA.3. Cancer 116(24):5599-5607. doi:10.1002/cncr.25393, PMID: 20824720

14. Crane CH, Varadhachary GR, Yordy JS et al (2011) Phase II trial of cetuximab, gemcitabine, and oxaliplatin followed by chemoradiation with cetuximab for locally advanced (T4) pancreatic adenocarcinoma: correlation of Smad4(Dpc4) immunostaining with pattern of disease progression. J Clin Oncol 29(22):3037-3043

15. Blackford A, Serrano OK, Wolfgang CL et al (2009) SMAD4 gene mutations are associated with poor prognosis in pancreatic cancer. Clin Cancer Res 15(14):4674 4679, PMID: 19584151

16. Kindler HL, Friberg G, Singh DA, Locker G, Nattam S, Kozloff M, Taber DA, Karrison T, Dachman A, Stadler WM, Vokes EE (2005) Phase II trial of bevacizumab plus gemcitabine in patients with advanced pancreatic cancer. J Clin Oncol 23(31):8033-8040

17. Kindler HL, Niedzwiecki D, Hollis D et al (2010) Gemcitabine plus bevacizumab compared with gemcitabine plus placebo in patients with advanced pancreatic cancer: phase III trial of the Cancer and Leukemia Group B (CALGB 80303). J Clin Oncol 28(22):36173622

18. Spano JP, Chodkiewicz C, Maurel J et al (2008) Efficacy of gemcitabine plus axitinib compared with gemcitabine alone in patients with advanced pancreatic cancer: an open-label randomised phase II study. Lancet 371(9630):2101-2108

19. Rajan A, Carter CA, Kelly RJ et al (2012) A phase I combination study of olaparib with cisplatin and gemcitabine in adults with solid tumors. Clin Cancer Res 18(8):2344-2351, PMID: 22371451

20. Tuli R, Surmak A, Hacker-Prietz A, Herman JM (2011) Poly (ADP ribose) polymerase-1/2 inhibition with veliparib enhances radiosensitization of pancreas cancer in vivo. Int J Radiat Oncol Biol Phys 81(2):S69-S70, ASTRO SS P-GI I Abstract

21. Wong J, Armour E, Kazanzides P, Iordachita I, Tryggestad E, Deng H, Matinfar M, Kennedy C, Liu Z, Chan T, Gray O, Verhaegen F, McNutt T, Ford E, DeWeese TL (2008) High-resolution, small animal radiation research platform with $\mathrm{x}$-ray tomographic guidance capabilities. Int J Radiat Oncol Biol Phys 71(5):1591-1599, PMID: 18640502

22. Tuli R, Surmak A, Reyes J, Hacker-Prietz A, Armour M, Leubner A, Blackford A, Tryggestad E, Jaffee EM, Wong J, Deweese TL, Herman JM (2012) Development of a novel preclinical pancreatic cancer 
research model: bioluminescence image-guided focal irradiation and tumor monitoring of orthotopic xenografts. Transl Oncol 5(2):77-84

23. Tuli R, Armour M, Surmak A, Reyes J, Iordachita I, Patterson M, Wong J (2013) Accuracy of off-line bioluminescence imaging to localize targets in preclinical radiation research. Radiat Res 179(4): 416-421, PMID: 23432050

24. Gause WC, Ekkens M, Nguyen D, Mitro V, Liu Q, Finkelman FD, Greenwald RJ, Urban JF (1999) The development of CD4 ${ }^{+}$T effector cells during the type 2 immune response. Immunol Res 20(1):55-65, PMID: 10467983

25. Staib L, Link KH, Beger HG (1999) Immunotherapy in pancreatic cancer - current status and future. Langenbeck's Arch Surg 384(4): 396-404

26. Argani P, Iacobuzio-Donahue C, Ryu B, Rosty C, Goggins M, Wilentz RE, Murugesan SR, Leach SD, Jaffee E, Yeo CJ, Cameron JL, Kern SE, Hruban RH (2001) Mesothelin is overexpressed in the vast majority of ductal adenocarcinomas of the pancreas: identification of a new pancreatic cancer marker by serial analysis of gene expression (SAGE). Clin Cancer Res 7(12):3862-3868

27. Weiss VL, Lee TH, Jaffee EM, Armstrong TD (2012) Targeting the right regulatory T-cell population for tumor immunotherapy. Oncoimmunology 1(7):1191-1193, PMID: 23170276

28. Weiss VL, Lee TH, Song $H$ et al (2012) Trafficking of high avidity HER-2/neu-specific T cells into HER-2/neu-expressing tumors after depletion of effector/memory-like regulatory T cells. PLoS One 7(2): e31962, PMID: 22359647

29. Le DT, Jaffee EM (2012) Regulatory T-cell modulation using cyclophosphamide in vaccine approaches a current perspective. Cancer Res 72(14):3439-3444, PMID: 22761338

30. Marrack P, Kappler J, Mitchell T (1999) Type I interferons keep activated T cells alive. J Exp Med 189(3):521-530

31. Matikainen S, Sareneva T, Ronni T, Lehtonen A, Koskinen PJ, Julkunen I (1999) Interferon-alpha activates multiple STAT proteins and upregulates proliferation-associated IL-2Ralpha, c-myc, and pim 1 genes in human T cells. Blood 93(6):1980-1991

32. Ma JH, Patrut E, Schmidt J, Knaebel HP, Buchler MW, Marten A (2005) Synergistic effects of interferon-alpha in combination with chemoradiation on human pancreatic adenocarcinoma. World $\mathrm{J}$ Gastroenterol 11(10):1521-1528

33. Schmidt J, Patrut E, Ma JH (2006) Immunomodulatory impact of interferon-alpha in combination with chemoradiation of pancreatic adenocarcinoma. Cancer Immunol Immunother 55(11):1396-1405

34. Pfeffer LM, Dinarello CA, Williams BR, Borden EC, Bordens R, Walter MR (1998) Biological properties of recombinant alphainterferons: 40th anniversary of the discovery of interferons. Cancer Res 58:2489-2499

35. Holsti LR, Mattson K, Niiranen A, Standertskiold-Nordenstam CG, Stenman S, Sovijarvi A (1987) Enhancement of radiation effects by alpha interferon in the treatment of small cell carcinoma of the lung. Int J Radiat Oncol Biol Phys 13:1161-1166

36. Takeuchi A, Kaneko S, Matsushita E, Urabe T, Shimoda A, Kobayashi K (1999) Interferon-alpha modulates resistance to cisplatin in three human hepatoma cell lines. J Gastroenterol 34:351-358

37. Hoffmann K, Mehrle S, Schmidt J, Büchler MW, Märten A (2008) Interferon-alpha restitutes the chemosensitivity in pancreatic cancer. Anticancer Res 28:1499-1508

38. Knaebel HP, Maerten A, Schmidt J, Hoffmann K, Seiler C, Lindel K et al (2005) Phase III trial of postoperative cisplatin interferon alpha$2 \mathrm{~b}$, and 5-FU combined with external radiation treatment versus 5FU alone for patients with resected pancreatic carcinoma - CapRI: study protocoll. BMC Cancer 5(1):37

39. Marten A, Schmidt J, Debus J et al (2010) CapRI: final results of the open-label, multicenter, randomized phase III trial of adjuvant chemoradiation plus interferon- $\alpha 2 b(\mathrm{CRI})$ versus 5 -FU alone for patients with resected pancreatic adenocarcinoma (PAC). J Clin Oncol 28: 18s, suppl; abstr LBA4012
40. Märten A, Schmidt J, Ose J, Harig S, Abel U, Münter MW, Jäger D, Friess H, Mayerle J, Adler G, Seufferlein T, Gress T, Schmid R, Büchler MW (2009) A randomized multicentre phase II trial comparing adjuvant therapy in patients with interferon alpha- $2 \mathrm{~b}$ and $5-\mathrm{FU}$ alone or in combination with either external radiation treatment and cisplatin (CapRI) or radiation alone regarding event-free survivalCapR. BMC Cancer 9:160

41. Lutz E, Yeo CJ, Lillemoe KD, Biedrzycki B, Kobrin B, Herman J, Sugar E, Piantadosi S, Cameron JL, Solt S, Onners B, Tartakovsky I, Choi M, Sharma R, Illei PB, Hruban RH, Abrams RA, Le D, Jaffee E, Laheru D (2011) A lethally irradiated allogeneic granulocytemacrophage colony stimulating factor-secreting tumor vaccine for pancreatic adenocarcinoma: a phase II trial of safety, efficacy, and immune activation. Ann Surg 253(2):328-335

42. Balmanoukian A, Ye X, Herman J, Laheru D, Grossman SA (2012) The association between treatment-related lymphopenia and survival in newly diagnosed patients with resected adenocarcinoma of the pancreas. Cancer Investig 30(8):571-576, PMID: 22812722

43. Herman JM, Chang DT, Goodman KA, et al. (2012) A phase II multi-institutional study to evaluate gemcitabine and fractionated stereotactic body radiotherapy for unresectable, locally advanced pancreatic adenocarcinoma. J Clin Oncol 30(suppl; abstract 4045)

44. Rwigema JC, Parikh SD, Heron DE et al (2011) Stereotactic body radiotherapy in the treatment of advanced adenocarcinoma of the pancreas. Am J Clin Oncol 34(1):63-69, PMID: 20308870

45. Nakamura M, Wada J, Suzuki H, Tanaka M, Katano M, Morisaki T (2009) Long-term outcome of immunotherapy for patients with refractory pancreatic cancer. Anticancer Res 29(3):831-836

46. Scalapino KJ, Daikh DI (2008) CTLA-4: a key regulatory point in the control of autoimmune disease. Immunol Rev 223:143-155, PMID: 18613834

47. Yy Z, Blank C, Gajewski TF (2004) Negative regulation of T-cell function by PD-1. Crit Rev Immunol 24(4):229-237, PMID: 15588223

48. Royal RE, Levy C, Turner K, Mathur A, Hughes M, Kammula US, Sherry RM, Topalian SL, Yang JC, Lowy I, Rosenberg SA (2010) Phase 2 trial of single agent ipilimumab (anti-CTLA-4) for locally advanced or metastatic pancreatic adenocarcinoma. J Immunother 33(8):828-833

49. Mole RH (1953) Whole body irradiation; radiobiology or medicine? Br J Radiol 26(305):234-241

50. Demaria $\mathrm{S}$ et al (2004) Ionizing radiation inhibition of distant untreated tumors (abscopal effect) is immune mediated. Int J Radiat Oncol Biol Phys 58:862-870

51. Dewan MZ, Galloway AE, Kawashima N, Dewyngaert JK, Babb JS, Formenti SC, Demaria S (2009) Fractionated but not single-dose radiotherapy induces an immune-mediated abscopal effect when combined with anti-CTLA-4 antibody. Clin Cancer Res 15(17): 5379-5388, PMID: 19706802

52. Camphausen K, Moses MA, Ménard C, Sproull M, Beecken WD, Folkman J, O'Reilly MS (2003) Radiation abscopal antitumor effect is mediated through p53. Cancer Res 63(8):1990-1993, PMID: 12702593

53. Watters D (1999) Molecular mechanisms of ionizing radiationinduced apoptosis. Immunol Cell Biol 77(3):263-271, PMID: 10361259

54. Lugade AA et al (2005) Local radiation therapy of B16 melanoma tumors increases the generation of tumor antigen-specific effector cells that traffic to the tumor. J Immunol 174:7516-7523

55. Frey B, Rubner Y, Wunderlich R, Weiss EM, Pockley AG, Fietkau R, Gaipl US (2012) Induction of abscopal anti-tumor immunity and immunogenic tumor cell death by ionizing irradiation-implications for cancer therapies. Curr Med Chem 19(12):1751-1764, 22414083 
56. Shiao SL, Ganesan AP, Rugo HS, Coussens LM (2011) Immune microenvironments in solid tumors: new targets for therapy. Genes Dev 25(24):2559-2572, PMID: 22190457

57. Cao ZA, Daniel D, Hanahan D (2002) Sub-lethal radiation enhances anti-tumor immunotherapy in a transgenic mouse model of pancreatic cancer. BMC Cancer 2:11, PMID: 12019035

58. Meng Y, Efimova EV, Hamzeh KW, Darga TE, Mauceri HJ, Fu YX, Kron SJ, Weichselbaum RR (2012) Radiation-inducible immunotherapy for cancer: senescent tumor cells as a cancer vaccine. Mol Ther 20(5):1046-1055, PMID: 22334019

59. Blanquicett C, Saif MW, Buchsbaum DJ, Eloubeidi M, Vickers SM, Chhieng DC, Carpenter MD, Sellers JC, Russo S, Diasio RB, Johnson MR (2005) Antitumor efficacy of capecitabine and celecoxib in irradiated and lead-shielded, contralateral human BxPC-3 pancreatic cancer xenografts: clinical implications of abscopal effects. Clin Cancer Res 11(24 Pt 1):8773-8781, PMID: 16361565 